pathways employed by shared cytokine receptors and thus is intermediate between broadly immunosuppressive glucocorticoids and individually targeted cytokine therapeutics.

Knowing which cytokine or cytokines to target in severe COVID-19 pneumonia remains a conundrum. One thing that is clearly emerging from this pandemic is that the CSS associated with COVID-19 is relatively unique, with only modestly elevated levels of IL-6, CRP and ferritin, for example. Studies exploring serum biomarkers clearly distinguish COVID-19induced CSS from more classic CSS such as macrophage-activation syndrome or hemophagocytic lymphohistiocytosis ${ }^{10}$. Conceivably, some of the relatively lower cytokine levels in patients with COVID-19 CSS may be related to the profound lymphopenia seen in these patients. By comparison, deadly strains of influenza virus, perhaps including the virus that caused the 1918 influenza pandemic, can lead to a more classic CSS ${ }^{11}$. Nevertheless, inhibition of the hyper-inflammatory immune response in severe COVID-19 pneumonia undoubtedly improves the survival of patients, particularly through glucocorticoids given to those with oxygen requirements. Timing of the administration of immunosuppressive or immunomodulatory agents is key-such that suppressing the host response too early during rapid viral replication is probably deleterious, whereas requirement for respiratory support may be the appropriate time to intervene. More-targeted approaches such as anakinra, however, may allow earlier introduction of anti-cytokine treatment.

As this pandemic stretches on, researchers and clinicians will continue to hone their knowledge of how best to treat patients hospitalized with severe COVID-19 pneumonia in order to improve survival rates. Current approaches include antivirals, proning and CSS therapies, among others. Beyond glucocorticoids, it remains to be seen precisely which targeted approaches are likely to be effective in treating the unique CSS induced by COVID-19. The finding of elevated levels of endogenous antagonist to the IL-1 receptor in patients with severe COVID-19 suggests that there is an IL-1 gene signature ${ }^{10}$. Perhaps IL-1 blockade with anakinra in select patients with COVID-19 pneumonia will gain traction as further data emerge. In the meantime, it is important to continue efforts to prevent the spread and development of severe SARS-CoV-2 infection with expanded availability and administration of safe and effective vaccines ${ }^{12}$.
Randy Q. Cron ${ }^{1 凶}$, Roberto Caricchio ${ }^{2}$ and W. Winn Chatham

${ }^{1}$ Division of Clinical Immunology and Rheumatology,

University of Alabama School of Medicine,

Birmingham, AL, USA. ${ }^{2}$ Section of Rheumatology,

Lewis Katz School of Medicine, Temple University,

Philadelphia, PA, USA.

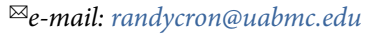

Published online: 3 September 2021

https://doi.org/10.1038/s41591-021-01500-9

References

1. Cron, R. Q. \& Chatham, W. W. J. Rheumatol. 47, 639-642 (2020).

2. Fajgenbaum, D. C. \& June, C. H. N. Engl. J. Med. 383,

2255-2273 (2020).

3. Beigel, J. H. et al. N. Engl. J. Med. 383, 1813-1826 (2020).

4. Horby, P. et al. N. Engl. J. Med. 384, 693-704 (2021).

5. Keller, M. J. et al. J. Hosp. Med. 15, 489-493 (2020).

6. Kyriazopoulou, E. et al. Nat. Med. https://doi.org/10.1038/s41591 021-01499-z (2021).

7. Caricchio, R. et al. J. Am. Med. Assoc. 326, 230-239 (2021).

8. Shankar-Hari, M. et al. J. Am. Med. Assoc. 326, 499-518 (2021).

9. Guimaraes, P. O. et al. N. Engl. J. Med. 385, 406-415 (2021)

10. Kessel, C. et al. Arthritis Rheumatol. https://doi.org/10.1002/ art.41763 (2021).

11. Schulert, G. S. et al. J. Infect. Dis. 213, 1180-1188 (2016)

12. Fauci, A. S. Science 372, 109 (2021).

Competing interests

R.Q.C. has served as a consultant to Sobi, Novartis, Pfizer and Sironax. R.Q.C. and W.W.C. have received grant support from Sobi for investigator-initiated clinical trials. R.C. has served as a consultant to GlaxoSmithKline, Johnson \& Johnson, Aurinia and Bristol Myers Squibb.

\title{
Anemia in women - an intractable problem that requires innovative solutions
}

\author{
A new spatial analysis calls for a tiered and geographically targeted approach to tackling the vast, ongoing burden \\ of anemia in women within low- and middle-income countries.
}

\section{Parul Christian}

\footnotetext{
A nemia in women of reproductive age (15-49 years of age) in low- and middle-income countries (LMICs) continues to be an intractable problem of 'hidden hunger', exemplifying gender health inequities and a shameful loss of human capital. The low hemoglobin concentration in blood that defines anemia occurs long after tissue iron stores have been depleted to levels associated with suboptimal function. A major cause of anemia, this iron deficiency leads to diminished oxygen-carrying capacity in red blood
}

cells, which in turn diminishes energy efficiency, work capacity and productivity ${ }^{1}$. In addition, severe anemia in pregnancy is consistently linked to maternal mortality ${ }^{2}$. Anemia in women of reproductive age is concentrated in LMICs and requires urgent and immediate attention. In this issue of Nature Medicine, Kinyoki and colleagues set out to model the prevalence and burden of mild, moderate and severe anemia in LMICs over the past two decades ${ }^{3}$.

The study examines data from several databases across 82 LMICs, comprising
218 geo-referenced household surveys conducted between 2000 and 2018 and including over three million women. On the basis of their analysis, the authors estimate half a billion women were living with anemia in LMICs in 2018. In the period from 2000 to 2018, they find limited decreases in anemia prevalence $(35.6 \%$ to $31.6 \%)$ and an increase in years lived with disability, due to population growth. They reveal widespread sub-national disparities and predict that a vast majority of countries will fail to achieve the World Health 


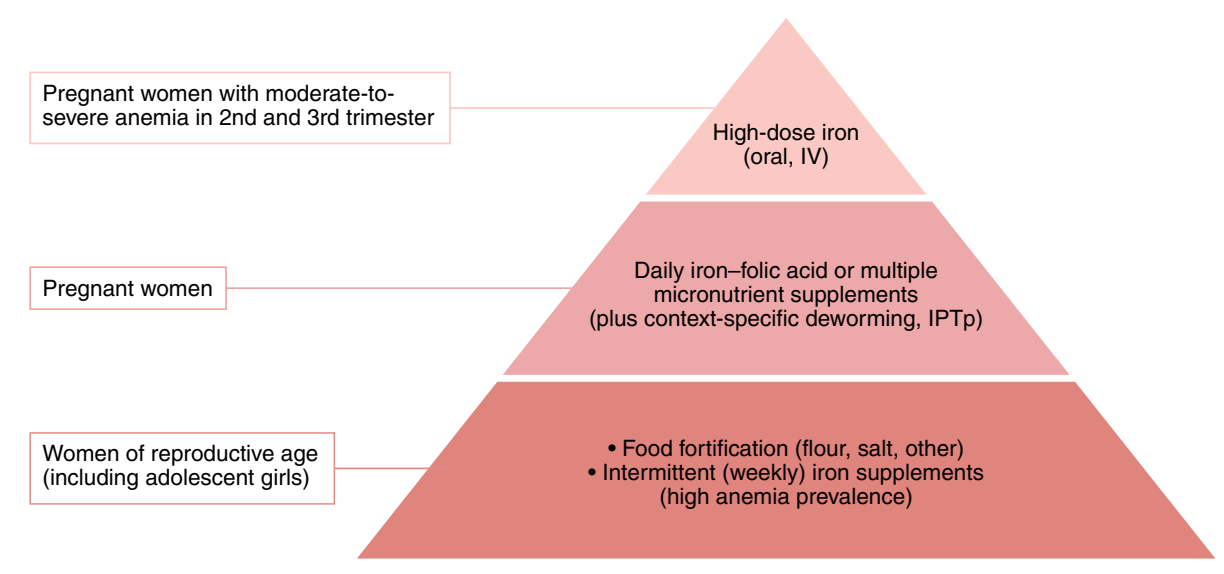

Fig. 1 | A life-course prevention-and-treatment approach to ameliorating anemia in women. IV, intravenous; IPTp, intermittent preventive treatment during pregnancy.

Organization global nutrition target of a $50 \%$ reduction in anemia prevalence by 2030. Although limited by data quality and quantity gaps, this novel analysis provides an opportunity to shed light, anew, on an important public-health problem and public-policy failure.

Physical productivity losses due to iron deficiency have been linked to the gross domestic product and potentially result in losses of billions of dollars annually ${ }^{4}$ (although more-robust and more-comprehensive estimates are needed). A recent analysis estimated gains of 2.28 million school years and US $\$ 8.25$ billion in lifetime income associated with scaling-up of antenatal iron-folic acid supplementation $^{5}$. An economic and human capital case is therefore well justified to convey the urgent need for programmatic action to policymakers and economic planners. India, with its high prevalence rate of $50 \%$, has the largest number of women of reproductive age with anemia (181.3 million) and harbors two thirds of the LMICs' cases ${ }^{3}$; clearly, it could accrue an enormous economic benefit by addressing this problem. Another 87 million women with anemia live in China, Pakistan and Nigeria.

Tackling this massive problem has been challenging because of issues such as gender inequality, failure of programs and reliance on economic development (and dietary change), which all stem in part from a general lack of political will. Etiologies such as malaria, soil-transmitted helminths and chronic inflammation do exist and may explain some of the geographic heterogeneity; however, iron deficiency and other nutritional causes of anemia predominate in women ${ }^{3}$ and may be linked to gender inequality in terms of access to a high-quality diet and, specifically, food sources rich in iron ${ }^{6}$. Highly bioavailable heme sources (meat, eggs and liver) are needed to meet the daily requirements for iron, which in women are set at $18 \mathrm{mg}$. One portion of three ounces ( 85 grams) of red meat provides $2.6 \mathrm{mg}$ of iron, one medium egg provides $0.6 \mathrm{mg}$ of iron, and a poorly absorbed plant source such as a cup (30 grams) of spinach provides only $0.8 \mathrm{mg}$ of iron. Consuming all three daily portions will meet only $22 \%$ of the recommended daily allowance for iron; the quality of diets for women in LMICs is much worse than this, and requirements are higher during pregnancy. Also, the recommended levels of intake would be insufficient to correct deficiency, particularly in a context in which $40-50 \%$ of the population is anemic. Linear programming has demonstrated that food-based strategies alone may not be sufficient to meet the dietary needs of women, calling for fortification and supplementation approaches instead ${ }^{7}$.

Antenatal iron-folic acid supplementation is an evidence-based, highly affordable (US\$1 per pregnancy) and recommended intervention. Nevertheless, large-scale programs have failed to achieve adequate coverage because of supply-chain breakdowns and low adherence. Political will and global momentum for maternal nutrition is lacking, with limited spending on maternal versus child health interventions ${ }^{8}$. Also, there is reliance on income growth for addressing the widely accepted drivers of change in anemia prevalence, such as female education, dietary improvement, and water, sanitation and hygiene ${ }^{9}$. However, anemia rates decrease only a quarter as fast as income increases ${ }^{10}$. Moreover, there is lack of data showing that behavior-change approaches for dietary diversification work, and there is evidence that interventions in water, sanitation and hygiene do not affect anemia ${ }^{11}$ (although both seem appropriate to do in the long run, and for their own sake). The slow decrease in anemia prevalence in women ${ }^{3}$ therefore calls for a different approach and a substantial commitment.

The analysis by Kinyoki et al. ${ }^{3}$ may help guide the future course of action. The type of resolute data generated, if used to create mapping and tracking tools, could be a powerful means for geographic targeting, resource allocation, deploying interventions and monitoring progress. In addition, a tiered, life-course approach (Fig. 1) may help to combat this problem. For the general population, a robust food-fortification effort is needed and has been proven to be effective for diminishing anemia in women of reproductive age ${ }^{12}$. Where the prevalence of anemia is high, fortification can be augmented with intermittent (weekly) iron supplementation as recommended by the World Health Organization. During pregnancy, supplementation with iron plus folic acid or, better still, with multiple micronutrients needs to be boosted through antenatal care and other platforms, alongside context-specific use of deworming and intermittent preventive treatment of malaria during pregnancy. Antenatal hemoglobin screening, which is recommended but poorly implemented, can be used in the context of treating the more severe forms of anemia with short-infusion, intravenously administered iron, as is practiced in high-income countries. If each tier in this strategy is effective, then the next tier will be smaller, as prevention is the goal.

There is an urgent need for more-accurate field-based diagnostics and point-of-care systems than the hemoglobinometers now used provide. Non-invasive technologies, including pulse oximetry, show some promise and greater acceptability, but their diagnostic performance needs improvement. Point-of-care systems for identifying underlying causes (iron deficiency, inflammation and hemoglobinopathy) are also needed as the field moves toward more-precise public-health prevention and treatment strategies. Much-needed work on redefining hemoglobin cut-offs for anemia seems to be underway, and treatment protocols also require updating. Treatment regimens with intravenous administration of low-molecular-weight iron products such as ferric carboxy maltose and ferric derisomaltose are promising but will require an affordable 'price point' for the LMIC context. The COVID-19 pandemic has exacerbated food and nutrition insecurity in 
LMICs and has done so disproportionately for women and children. Maternal anemia is a scourge that cannot exist in such proportions and calls for immediate, large-scale equity-based and economically justified action.

Parul Christian $ه$

Department of International Health, Center for Human Nutrition, Johns Hopkins Bloomberg School of Public Health, Baltimore, MD, USA.

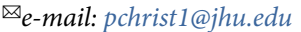

Published online: 12 October 2021

https://doi.org/10.1038/s41591-021-01514-3

References

1. Haas, J. D. \& Brownlie, T. IV I. Nutr. 131, 676S-690S (2001).

2. Christian, P. in Handbook of Nutrition and Pregnancy 2nd edn. (eds. Lammi-Keefe, C.J., Couch, S. \& Kirwan, J.P.) 401-421 (Humana Press, 2018).

3. Kinyoki, D. et al. Nat. Med. https://doi.org/10.1038/s41591-02101498-0 (2021).

4. Horton, S. \& Ross, J. Food Policy 28, 51-75 (2003).

5. Perumal, N. et al. Am. J. Clin. Nutr. https://doi.org/10.1093/ajcn/ nqab234 (2021)
6. Sedlander, E et al Nutrition 86, 111159 (2021).

7. Ferguson, E. L. et al. Matern. Child Health J. 23, 55-66 (2019).

8. Christian, P., Smith, E. R. \& Zaidi, A. BMJ Glob. Health $\mathbf{5}$ e002186 (2020).

9. Nguyen, P. H., Scott, S., Avula, R., Tran, L. M. \& Menon, P. BMJ Glob. Health 3, e001010 (2018).

10. Alderman, H. \& Linnemayr, S. Food Nutr. Bull. 30, 265-269 (2009).

11. Baldi, A. J., Clucas, D. \& Pasricha, S. R. Am. J. Clin. Nutr. 112, 1145-1146 (2020).

12. Keats, E. C., Neufeld, L. M., Garrett, G. S., Mbuya, M. N. N. \& Bhutta, Z. A. Am. J. Clin. Nutr. 109, 1696-1708 (2019).

Competing interests

The author declares no competing interests.

\title{
Empowering prosthesis users with a hip exoskeleton
}

\author{
A phase 1 trial shows the feasibility of a novel approach combining a powered hip exoskeleton with a passive leg \\ prosthesis to improve the energy efficiency of walking.
}

Varun Nalam and He (Helen) Huang

T he advent of wearable assistive technology has never been more apparent than in the development of lower limb prosthetics for locomotion. These have evolved from passive devices to fully powered robotic devices capable of replicating the complete functionality of the missing limb. However, robotic prosthesis technology is still in its infancy, and for the time being, passive devices are predominantly used in the clinic. Much research in the field is aimed at reducing the weight of robotic prostheses and advancing robotic prosthesis function through the development of more intuitive controllers that are capable of performing a wider range of tasks in different environments. In this issue of Nature Medicine, Ishmael et al. take a novel approach: instead of advancing the technology of lower limb prostheses, the authors engineered an exoskeleton at the hip joint of the residual limb in conjunction with a passive prosthesis, and showed a significant improvement in walking economy relative to a passive prosthesis alone ${ }^{1}$. This approach centers on the residual joints of transfemoral amputees and leverages the recent advancements in powered exoskeletons ${ }^{1}$, which are often applied to healthy persons or to individuals with neuromotor deficits (Fig. 1). By doing so, this study opens up a new and exciting pathway for wearable assistive technology for the amputee population.

Understanding the biomechanics of people with a transfemoral amputation and the effects of passive prostheses can provide insight into the different paths of research in the field. Human locomotion involves coordination and power generation at the three major joints of the lower limb, with most of the propulsive power being generated by the hip and ankle joints ${ }^{2}$. The current passive prostheses used by transfemoral amputees do not provide power at the ankle and knee. In addition, many residual thigh muscles that originally functioned around the hip joint go on to lose their force production capability due to transfemoral amputations. This leads to a weak residual hip joint on amputated limbs. Hence, amputees rely on the weak residual hip and on compensation from the contralateral intact limb and trunk for daily task performance-which leads to gait asymmetry ${ }^{3}$, reduced walking stability ${ }^{4}$ and secondary physical conditions ${ }^{5}$ (such as osteoarthritis and back pain). New solutions are therefore needed to improve the function, long-term health and quality of life of individuals with transfemoral amputations.

To address these issues, powered knee or knee-ankle prostheses with improved design and control have been developed ${ }^{6}$, and some-including a powered knee system (the PowerKnee from Össur)-are already clinically available. These powered prostheses provide net positive power in the prosthesis joint to replace the power previously generated by lower limb muscles, and they have been shown to generate near-normative knee and ankle mechanics and improved balance confidence ${ }^{7}$. In addition, these devices enable amputees to navigate stairs, negotiate ramps and perform sit-to-stand transition, all of which are difficult for amputees wearing passive devices. However, limitations include a lack of somatosensory feedback as well as control challenges in coordinating with the user's intent, all of which compromise the efficient energy transfer from powered prosthesis to amputee user for improved walking economy ${ }^{8}$. Moreover, the mechanisms required for powered prosthesis add additional weight on the distal end of the foot, causing discrepancies in weight distribution and increased loading on the residual hip, which in turn increases the (sense of) effort required for walking.

In comparison, the concept of the study by Ishmael et al..$^{1}$ was to assist the weak residual hip joint on the amputated side using a powered hip exoskeleton to produce the necessary hip torque and alleviate the 